\title{
Erratum to: Poster Abstracts
}

Published online: 18 January 2012

(C) Springer Science+Business Media, LLC 2012

Erratum to: J Mol Neurosci (2010) 40:236-265

DOI 10.1007/s12031-009-9277-5

In the original version of this article, a sponsor was unfortunately omitted under A BIMODAL ROLE FOR ACHE IN REGULATING LYMPHOCYTIC PROLIFERATION (J. Mol. Neuroscience. 2010, 40:240-245) section.

We ask to add to the Acknowledgment section in the following: "C. Perry was supported by a research fellowship grant from the Israeli Ministry of Health". 